\title{
Scattering paths in test optical bench for LISA mission
}

\author{
Marco Nardello ${ }^{a}$, Michel Lintz ${ }^{a}$, Christelle Buy ${ }^{b}$ (on behalf of the LISA-France collaboration) \\ a ARTEMIS, OCA, Université Cote d'Azur and CNRS, 96 Boulevard de l'Observatoire, 06300 Nice, France \\ b L2IT - Université Paul Sabatier 31062 Toulouse, France \\ marco.nardello@oca.eu \\ michel.lintz@oca.eu \\ christelle.buy@l2it.in2p3.fr
}

\begin{abstract}
Scattered light can disrupt heterodyne measurements in the ZIFO test bench for LISA mission. This study shows a method to find all possible scattering paths and calculate their power, confirming the feasibility of the system. (C2020 The Author(s)
\end{abstract}

\section{Introduction}

Foreseen to be launched in 2034, LISA (Laser Interferometer Space Antenna) is ESA's L3 mission. It will detect gravitational waves thanks to a constellation of 3 spacecraft separated by 2.5 million km in a triangular formation, following Earth in its orbit around the Sun. The separation of free-floating reference surfaces in each satellite is measured precisely using laser interferometry, in order to measure minuscule variations caused by a passing gravitational wave [1].

A high precision heterodyne interferometry (ZIFO) optical bench is under development, featuring some of the foreseen complexity and uncertain technologies dictated by LISA instrumentation [2]. It is developed by a collaboration of French laboratories within the LISA France consortium, and will be used to demonstrate the capability to solve the technical challenges imposed by LISA requirements in term of high precision optical metrology, low noise acquisition chain, environment control, etc.

One stringent requirement imposed by the mission is the very low level of phase noise, that must not exceed $1 \mu$ cycle [3]. This means that stray light power must be kept to a controlled level to obtain sensible measurement.

In the following is shown a procedure adopted to find possible scattered light sources and paths on an arbitrarily complex optical system (e.g. the ZIFO), and to calculate the resulting stray light power on any interferometer.

\section{ZIFO bench description}

Figure 1 shows the optical design for which stray light calculations were performed:

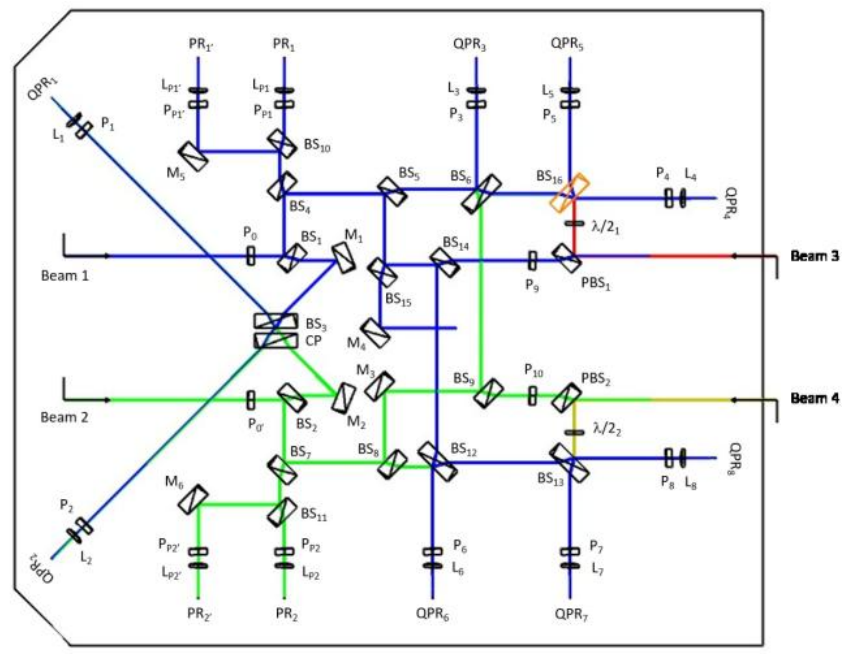

Fig. 1. ZIFO optical design.

Two laser beams (Beam 1 and Beam 2) are injected in the system and directed towards 8 quadrant interferometers (QPRs), simulating LISA heterodyne signals, and 4 reference photodiodes (PRs). To manipulate the lasers, 16 beam splitters, 2 polarizing beam splitters, 6 mirrors, 12 polarizers, 8 lenses and 2 half wave plates are present. Each optical element being a potential stray light source. 


\section{Scattering paths}

In a system with a so complex geometry is not easy to guess which paths will contribute to scattering. To find all the relevant scattering directions we had to resort to back raytracing with FRED software. We put virtual sources in correspondence of each QPR and traced light back to the optical elements, obtaining a list of all the positions and directions of arrival on a scattering surface. Capability to generate interference was also a main concern in choosing the characteristic of rays to be created. In Figure 2 results are shown concerning beam splitter BS6:

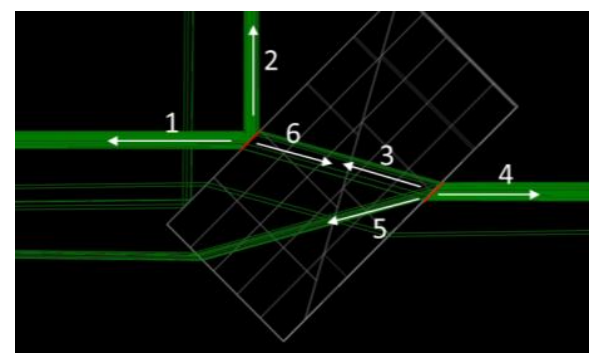

Fig. 2 Back raytracing from QPDs to BS6

Green rays, originated on a detector and having been deflected by the other optical elements, arrive on the red areas on BS6, so each and only the directions indicated by them (and by white arrows) are possible scattering paths.

\section{Results}

On FRED software path directions were used to calculate scattering power on detectors, for different roughness (1 or $10 \mathrm{~nm}$ ) and contamination levels (CL300 and 600), representing typical values and worst-case scenario. For polarizers empirical data were at disposal and have been used.

Table 1 lists the scattering power ratio with respect to nominal beam on each QPD, due to the sum of contributions from each optical element present on the bench.

Table 1. Scattering power ratios

\begin{tabular}{ccccc}
\hline Detector & $1 \mathrm{~nm}$ & $10 \mathrm{~nm}$ & CL300 & CL600 \\
\hline QPR1 & $8.0 \mathrm{E}-10$ & $2.2 \mathrm{E}-8$ & $2.4 \mathrm{E}-9$ & $5.4 \mathrm{E}-8$ \\
QPR2 & $8.0 \mathrm{E}-10$ & $2.2 \mathrm{E}-8$ & $2.4 \mathrm{E}-9$ & $5.4 \mathrm{E}-8$ \\
QPR3 & $4.6 \mathrm{E}-11$ & $1.2 \mathrm{E}-9$ & $1.3 \mathrm{E}-10$ & $3.0 \mathrm{E}-9$ \\
QPR4 & $5.7 \mathrm{E}-11$ & $3.0 \mathrm{E}-9$ & $5.5 \mathrm{E}-10$ & $1.5 \mathrm{E}-8$ \\
QPR5 & $6.1 \mathrm{E}-11$ & $3.3 \mathrm{E}-9$ & $6.1 \mathrm{E}-10$ & $1.7 \mathrm{E}-8$ \\
QPR6 & $1.9 \mathrm{E}-10$ & $2.0 \mathrm{E}-9$ & $3.3 \mathrm{E}-10$ & $4.9 \mathrm{E}-9$ \\
QPR7 & $1.8 \mathrm{E}-10$ & $6.4 \mathrm{E}-9$ & $1.3 \mathrm{E}-9$ & $3.4 \mathrm{E}-8$ \\
QPR8 & $1.7 \mathrm{E}-10$ & $5.9 \mathrm{E}-9$ & $1.1 \mathrm{E}-9$ & $3.0 \mathrm{E}-8$ \\
\hline
\end{tabular}

Results show that contamination will be a bigger factor than surface roughness, concerning stray light. The power level here reported are low enough to allow a correct operation of the system.

\section{Conclusions}

The search for scattering paths in the IFO bench confirmed the feasibility of the system. All scattering paths were explored and the amount of stray light power reaching the detector remains at level that does not influence heterodyne measurements in a disruptive way.

\section{Acknowledgements}

This work and the development of the ultra-stable optical bench are performed by the LISA France collaboration and funded by the French Space Agency (CNES)

\section{References}

[1] P. Amaro-Seoane, H. Audley, S. Babak et al., "Laser Interferometer Space Antenna" arXiv:1702.00786 (2017)

[2] H. Halloin, " Development of Optical Demonstrators for the Integration of the LISA Instrument ", submitted to XIII LISA Symposium (2020)

[3] H. Halloin, "Stray light to phase coupling", LISA internal technical note LISA-APC-INST-TN-005 v1.2, 10/07/2019. 